\title{
Ligand-mediated nanocluster formation with classical and autocatalytic growth
}

\author{
Mohsen Farshad, ${ }^{\ddagger}$ Dylan Suvlu, ${ }^{\ddagger}$ and Jayendran C. Rasaiah* \\ Department of Chemistry, University of Maine, Orono, Maine 04469, United States
}

Supporting Information

\section{List of Symbols}

$k_{p, 1} \quad$ Monomer formation rate coefficient, $\mathrm{s}^{-1}$

$k_{p, 2} \quad$ Reduction of ligand-associated monomer ion rate coefficient, $\mathrm{s}^{-1}$

$k_{b, 1} \quad$ Ligand binding to metal atom rate coefficient, $\mathrm{M}^{-1} \mathrm{~s}^{-1}$

$k_{u b, 1} \quad$ Ligand unbinding to metal atom rate coefficient, $\mathrm{s}^{-1}$

$k_{b, 2} \quad$ Ligand binding to metal ion rate coefficient, $\mathrm{M}^{-1} \mathrm{~s}^{-1}$

$k_{u b, 2} \quad$ Ligand unbinding to metal ion rate coefficient, $\mathrm{s}^{-1}$

$k_{n} \quad$ Self-dimerization rate coefficient, $\mathrm{M}^{-1} \mathrm{~s}^{-1}$

$k_{n, a c} \quad$ Autocatalytic dimerization rate coefficient, $\mathrm{M}^{-1} \mathrm{~s}^{-1}$

$k_{g} \quad$ Monomer addition growth rate coefficient, $\mathrm{M}^{-1} \mathrm{~s}^{-1}$

$k_{d} \quad$ Monomer dissociation rate coefficient, $\mathrm{s}^{-1}$

$k_{g, a c} \quad$ Autocatalytic growth rate coefficient, $\mathrm{M}^{-1} \mathrm{~s}^{-1}$

$k_{d, a c} \quad$ Autocatalytic dissociation rate coefficient, $\mathrm{s}^{-1}$

$k_{a} \quad$ Ligand association rate coefficient, $\mathrm{M}^{-1} \mathrm{~s}^{-1}$

$k_{e} \quad$ Ligand elimination rate coefficient, $\mathrm{s}^{-1}$

$t \quad$ Time of reaction, $s$

$i \quad$ Number of monomers, - 
j Number of ligands, -

$\left[\mathrm{M}^{+}\right] \quad$ Concentration of metal ion, $\mathrm{M}$

[M] Concentration of metal atom, M

[L] Concentration of Ligand, M

[ML] Concentration of ligand associated metal atom, M

$\left[\mathrm{ML}^{+}\right] \quad$ Concentration of ligand associated metal ion, $\mathrm{M}$

$\left[\mathrm{C}_{2, j}\right] \quad$ Concentration of dimer with $j$ ligands, $\mathrm{M}$

$\left[\mathrm{C}_{i, j}\right] \quad$ Concentration of cluster with $i$ monomers and $j$ ligands, $\mathrm{M}$

$\left[\overline{\mathrm{C}}_{i}\right] \quad$ Concentration of cluster with $i$ monomers regardless of number of $j$ ligands, M

$\left[\overline{\mathrm{L}}_{i}\right] \quad$ First order moment, $\mathrm{M}$

$\left[\overline{\mathrm{L}}_{i}\right] \quad$ Second order moment, $\mathrm{M}$

$N_{s, i} \quad$ Number of sites on a cluster with $i$ monomers, -

$p(j \mid i) \quad$ probability of finding $j$ ligands on a given cluster of $i$ monomers, -

$p_{i} \quad$ probability of finding a bound ligand with on a cluster with $i$ monomers, -

$\mathrm{C}(i, t) \quad$ Monomeric concentration of clusters with $i$ monomers at time $t, \mathrm{M}$

$\mathrm{C}(D, t) \quad$ Concentration of cluster with $i$ monomers at time $t, \mathrm{M}$

$D_{M} \quad$ Monomer diameter, $\mathrm{m}$

Here we present the rate equations in our model before and after applying method of moments. ${ }^{1}$ The reaction scheme is reproduced below: 
$\mathbf{M}^{+} \stackrel{k_{p, 1}}{\longrightarrow} \mathrm{M} ;$ Monomer formation

$\mathrm{M}+\mathrm{L} \underset{k_{u b, 1}}{\stackrel{k_{b, 1}}{\rightleftharpoons}} \mathrm{ML} ;$ Ligand association with metal atom

$\mathrm{M}^{+}+\mathrm{L} \underset{k_{u b, 2}}{\stackrel{k_{b, 2}}{\rightleftharpoons}} \mathrm{ML}^{+}$; Ligand association with metal ions

$\mathrm{ML}^{+} \stackrel{k_{p, 2}}{\longrightarrow} \mathrm{ML} ;$ Monomer formation

$\mathrm{ML}+\mathrm{ML} \stackrel{k_{n}}{\longrightarrow} \mathrm{C}_{2,2} ;$ Self-dimerization

$\mathrm{ML}+\mathrm{ML}^{+} \stackrel{k_{n, a c}}{\longrightarrow} \mathrm{C}_{2,2} ;$ Autocatalytic dimerization

$\mathrm{C}_{i, j}+\mathrm{ML} \underset{k_{d, j+1}}{\stackrel{k_{g, i, j}}{\rightleftharpoons}} \mathrm{C}_{i+1, j+1} ;$ Monomer addition growth

$\mathrm{C}_{i, j}+\mathrm{ML}^{+} \underset{k_{d, j+1, a c}}{\stackrel{k_{g, i, j a c}}{\rightleftharpoons}} \mathrm{C}_{i+1, j+1} ;$ Autocatalytic growth

$\mathrm{C}_{i, j}+\mathrm{L} \underset{k_{e, j+1}}{\stackrel{k_{a, i j}}{\rightleftharpoons}} \mathrm{C}_{i, j+1} ;$ Ligand association/elimination to cluster

The minimum value of $i$ is 2 and the maximum was set to 400 in calculations. The rate equations before applying method of moments of the above model are as follows:

$$
\begin{aligned}
& \frac{d\left[\mathrm{M}^{+}\right]}{d t}=k_{p, 1}\left[\mathrm{M}^{+}\right] \quad k_{b, 1}\left[\mathrm{M}^{+}\right][\mathrm{L}]+k_{u b, 1}\left[\mathrm{ML}^{+}\right] \\
& \frac{d[\mathrm{M}]}{d t}=k_{p, 1}\left[\mathrm{M}^{+}\right] \quad k_{b, 2}[\mathrm{M}][\mathrm{L}]+k_{u b, 2}[\mathrm{ML}] \\
& \frac{d[\mathrm{~L}]}{d t}=k_{b, 1}[\mathrm{M}][\mathrm{L}]+k_{u b, 1}[\mathrm{ML}] \quad k_{b, 2}\left[\mathrm{M}^{+}\right][\mathrm{L}]+k_{u b, 2}\left[\mathrm{ML}^{+}\right] \\
& k_{a}[\mathrm{~L}]{ }_{i=2}^{i_{\max } N_{s, i}}\left[\mathrm{C}_{i, j}\right]\left(N_{s, i} \quad j\right)+k_{e}{ }_{i=2}^{i_{\max } N_{s, i}}\left[\mathrm{C}_{i, j}\right] j \\
& \frac{d[\mathrm{ML}]}{d t}=k_{p, 2}\left[\mathrm{ML}^{+}\right]+k_{b, 1}[\mathrm{M}][\mathrm{L}] \quad k_{u b, 1}[\mathrm{ML}] \quad 2 k_{n}[\mathrm{ML}]^{2} \quad k_{n, a c}[\mathrm{ML}]\left[\mathrm{ML}^{+}\right] \\
& k_{g}[\mathrm{ML}]_{i_{\max } N_{s, i}}^{N_{i=2}}\left[\mathrm{C}_{i, j}\right]\left(N_{s, i} \quad j\right)+k_{d}^{i_{\max } N_{s, i}}\left[\mathrm{C}_{i, j}\right] j
\end{aligned}
$$




$$
\begin{aligned}
& \frac{d\left[\mathrm{ML}^{+}\right]}{d t}=k_{b, 2}\left[\mathrm{M}^{+}\right][\mathrm{L}] \quad k_{u b, 2}\left[\mathrm{ML}^{+}\right] \quad k_{n, a c}[\mathrm{ML}]\left[\mathrm{ML}^{+}\right] \\
& k_{g, a c}\left[\mathrm{ML}^{+}\right]{ }_{i=2}^{i_{\max } N_{s, i}}\left[\mathrm{C}_{i, j}\right]\left(N_{s, i} j\right)+k_{d, a c}{ }_{i=3}^{i_{\max } N_{s, i}}\left[\mathrm{C}_{i, j}\right] j \\
& \frac{d}{d t}\left[\mathrm{C}_{2,2}\right]=k_{n}[M L]^{2}+k_{n, a c}[\mathrm{ML}]\left[\mathrm{ML}^{+}\right]-\left(k_{g}[\mathrm{ML}]+k_{g, a c}\left[\mathrm{ML}^{+}\right]\right)\left[\mathrm{C}_{2,2}\right]\left(N_{s, 2}-2\right) \\
& +\left(k_{d}+k_{d, a c}\right) 3\left[\mathrm{C}_{3,3}\right]-k_{a}[L]\left\{\left[\mathrm{C}_{2,2}\right]\left(N_{s, 2}-2\right)-\left[\mathrm{C}_{2,1}\right]\left(N_{s, 2}-1\right)\right\} \\
& +k_{e}\left\{3\left[\mathrm{C}_{2,3}\right]-2\left[\mathrm{C}_{2,2}\right]\right\} \\
& \frac{d}{d t}\left[\mathrm{C}_{i, j}\right]=-\left(k_{g}[\mathrm{ML}]+k_{g, a c}\left[\mathrm{ML}^{+}\right]\right)\left\{\left[\mathrm{C}_{i, j}\right]\left(N_{s, i}-j\right)-\left[\mathrm{C}_{i-1, j-1}\right]\left(N_{s, i-1}-j+1\right)\right\} \\
& +\left(k_{d}+k_{d, a c}\right)\left\{\left[\mathrm{C}_{i+1, j+1}\right](j+1)-\left[\mathrm{C}_{i, j}\right] j\right\}-k_{a}[L]\left\{\left[\mathrm{C}_{i, j}\right]\left(N_{s, i}-j\right)-\left[\mathrm{C}_{i, j-1}\right]\left(N_{s, i}-j+1\right)\right\} \\
& +k_{e}\left\{\left[\mathrm{C}_{i, j+1}\right](j+1)-\left[\mathrm{C}_{i, j}\right] j\right\} \quad 3 \leq i \leq 400
\end{aligned}
$$

As described in the main text, we used method of moments to convert the equations with two internal coordinates (2-D) to two one internal coordinates (1-D). The full set of equations used for calculations are as follows

$$
\begin{aligned}
& \frac{d\left[\mathrm{M}^{+}\right]}{d t}=k_{p, 1}\left[\mathrm{M}^{+}\right] \quad k_{b, 1}\left[\mathrm{M}^{+}\right][\mathrm{L}]+k_{u b, 1}\left[\mathrm{ML}^{+}\right] \\
& \frac{d[\mathrm{M}]}{d t}=k_{p, 1}\left[\mathrm{M}^{+}\right] \quad k_{b, 2}[\mathrm{M}][\mathrm{L}]+k_{u b, 2}[\mathrm{ML}] \\
& \frac{d[\mathrm{~L}]}{d t}=-k_{b, 1}[\mathrm{M}][\mathrm{L}]+k_{u b, 1}[\mathrm{ML}]-k_{b, 2}\left[\mathrm{M}^{+}\right][\mathrm{L}]+k_{u b, 2}\left[\mathrm{ML}^{+}\right] \\
& -k_{a}[\mathrm{~L}] \sum_{i=2}^{i_{\max }}\left\{\left[\overline{\mathrm{C}}_{i}\right] N_{s, i}-\left[\overline{\mathrm{L}}_{i}\right]\right\}+k_{e} \sum_{i=2}^{i_{\max }}\left[\overline{\mathrm{L}}_{i}\right] \\
& \frac{d[\mathrm{ML}]}{d t}=k_{p, 2}\left[\mathrm{ML}^{+}\right]+k_{b, 1}[\mathrm{M}][\mathrm{L}]-k_{u b, 1}[\mathrm{ML}]-2 k_{n}\left[\mathrm{ML}^{2}-k_{n, a c}\left[\mathrm{ML}^{2}\right]\left[\mathrm{ML}^{+}\right]\right. \\
& -k_{g}[\mathrm{ML}] \sum_{i=2}^{i_{\max }}\left\{\left[\overline{\mathrm{C}}_{i}\right] N_{s, i}-\left[\overline{\mathrm{L}}_{i}\right]\right\}+k_{d} \sum_{i=3}^{i_{\max }}\left[\overline{\mathrm{L}}_{i}\right] \\
& \frac{d[\mathrm{ML}]}{d t}=-k_{p, 2}\left[\mathrm{ML}^{+}\right]+k_{b, 2}\left[\mathrm{M}^{+}\right][\mathrm{L}]-k_{u b, 2}\left[\mathrm{ML}^{+}\right]-k_{n, a c}[\mathrm{ML}]\left[\mathrm{ML}^{+}\right] \\
& -k_{g, a c}\left[\mathrm{ML}{ }^{+}\right] \sum_{i=2}^{i_{\max }}\left\{\left[\overline{\mathrm{C}}_{i}\right] N_{s, i}-\left[\overline{\mathrm{L}}_{i}\right]\right\}+k_{d, a c} \sum_{i=3}^{i_{\max }}\left[\overline{\mathrm{L}}_{i}\right]
\end{aligned}
$$




$$
\begin{aligned}
& \frac{d}{d t}\left[\overline{\mathrm{C}}_{2}\right]=k_{n}[\mathrm{ML}]^{2}+k_{n, a c}[\mathrm{ML}]\left[\mathrm{ML}^{+}\right]-\left(k_{g}[\mathrm{ML}]+k_{g, a c}\left[\mathrm{ML}^{+}\right]\right)\left\{\left[\overline{\mathrm{C}}_{2}\right] N_{s, 2}-\left[\overline{\mathrm{L}}_{2}\right]\right\} \\
& +\left(k_{d}+k_{d, a c}\right)\left[\overline{\mathrm{L}}_{3}\right] \\
& \frac{d}{d t}\left[\overline{\mathrm{L}}_{2}\right]=k_{n}[\mathrm{ML}]^{2}+k_{n, a c}[\mathrm{ML}]\left[\mathrm{ML}^{+}\right]-\left(k_{g}[\mathrm{ML}]+k_{g, a c}\left[\mathrm{ML}^{+}\right]\right)\left\{\left[\overline{\mathrm{L}}_{2}\right] N_{s, 2}-\left[\overline{\overline{\mathrm{L}}}_{2}\right]\right\} \\
& +\left(k_{d}+k_{d, a c}\right)\left\{\left[\overline{\mathrm{L}}_{3}\right]-\left[\overline{\mathrm{L}}_{3}\right]\right\}-k_{a}[\mathrm{~L}]\left\{\left[\overline{\mathrm{C}}_{2}\right] N_{s, 2}-\left[\overline{\mathrm{L}}_{2}\right]\right\}-k_{e}\left[\overline{\mathrm{L}}_{2}\right] \\
& \frac{d}{d t}\left[\overline{\mathrm{C}}_{i}\right]=-\left(k_{g}[\mathrm{ML}]+k_{g, a c}\left[\mathrm{ML}^{+}\right]\right)\left\{\left(\left[\overline{\mathrm{C}}_{i}\right] N_{s, i}-\left[\overline{\mathrm{L}}_{i}\right]\right)-\left(\left[\overline{\mathrm{C}}_{i-1}\right] N_{s, i-1}-\left[\overline{\mathrm{L}}_{i-1}\right]\right)\right\} \\
& +\left(k_{d}+k_{d, a c}\right)\left\{\left[\overline{\mathrm{L}}_{i+1}\right]-\left[\overline{\mathrm{L}}_{i}\right]\right\} \quad 3 \leq i \leq 400 \\
& \frac{d}{d t}\left[\overline{\mathrm{L}}_{i}\right]=-\left(k_{g}[\mathrm{ML}]+k_{g, a c}\left[\mathrm{ML}^{+}\right]\right)\left\{\left(\left[\overline{\mathrm{L}}_{i}\right] N_{s, i}-\left[\overline{\mathrm{L}}_{i}\right]\right)-\left(\left[\overline{\mathrm{C}}_{i-1}\right] N_{s, i-1}+\left[\overline{\mathrm{L}}_{i-1}\right]\left(N_{s, i-1}-1\right)-\left[\overline{\mathrm{L}}_{i-1}\right]\right)\right\} \\
& +\left(k_{d}+k_{d, a c}\right)\left\{\left(\left[\overline{\mathrm{L}}_{i+1}\right]-\left[\overline{\mathrm{L}}_{i+1}\right]\right)-\left[\overline{\mathrm{L}}_{i}\right]\right\}-k_{a}[\mathrm{~L}]\left\{\left[\overline{\mathrm{C}}_{i}\right] N_{s, i}-\left[\overline{\mathrm{L}}_{i}\right]\right\}-k_{e}\left[\overline{\mathrm{L}}_{i}\right] \quad 3 \leq i \leq 400
\end{aligned}
$$

We solved the equations numerically using ode15s solver in MATLAB. The solution to the equations provides the concentration of clusters with $i$ monomers with average concentration of ligands on clusters with $i$ monomers in time $t$. We convert the monomeric concentration of clusters to the concentration of clusters having a diameter $D$ as described in the main text. Table $\mathrm{S} 1$ presents diameter intervals taken from Malvern Zetasizer Nano ZS instrument to create histograms using Eq. S19, S20.

$$
\begin{gathered}
C_{\text {hist }}(t)=\frac{\sum_{D_{1}<D<D_{2}} C_{D}(t)}{\sum_{i} C_{D}(t)} \\
D_{\text {hist }}=\frac{\sum_{D_{1}<D<D_{2}} D C_{D}}{\sum_{D} C_{D}(D)}
\end{gathered}
$$

Table S1. Diameter intervals used in histograms.

\begin{tabular}{cc}
\hline $\boldsymbol{D}_{\mathbf{1}}(\mathbf{n m})$ & $\boldsymbol{D}_{\mathbf{2}}(\mathbf{n m})$ \\
\hline 0.000 & 0.400 \\
0.400 & 0.463 \\
0.463 & 0.536 \\
0.536 & 0.621 \\
0.621 & 0.719 \\
\hline
\end{tabular}




\begin{tabular}{ll}
\hline 0.719 & 0.833 \\
0.833 & 0.965 \\
0.965 & 1.117 \\
\hline
\end{tabular}

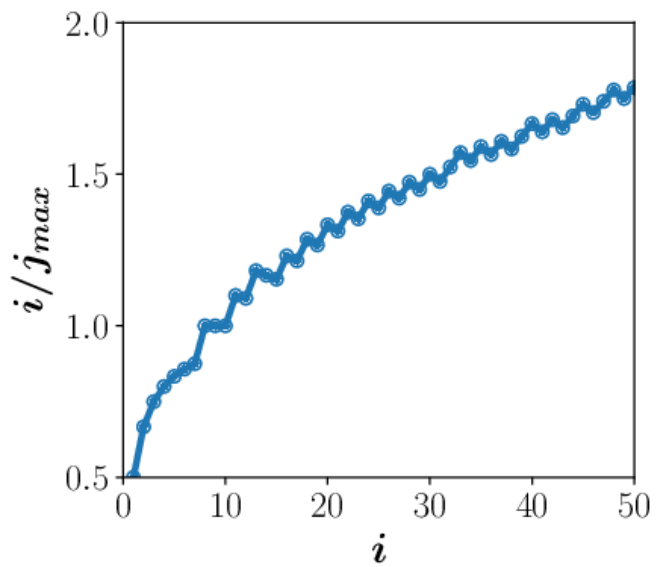

Figure S1: Ratio of metal atoms, $i$, to the number of ligand binding sites $j_{\max }$ (defined by $N_{s, i}$ ) as a function of the number of metal atoms in the cluster. As the figure indicates, the ratio increases with $i$.
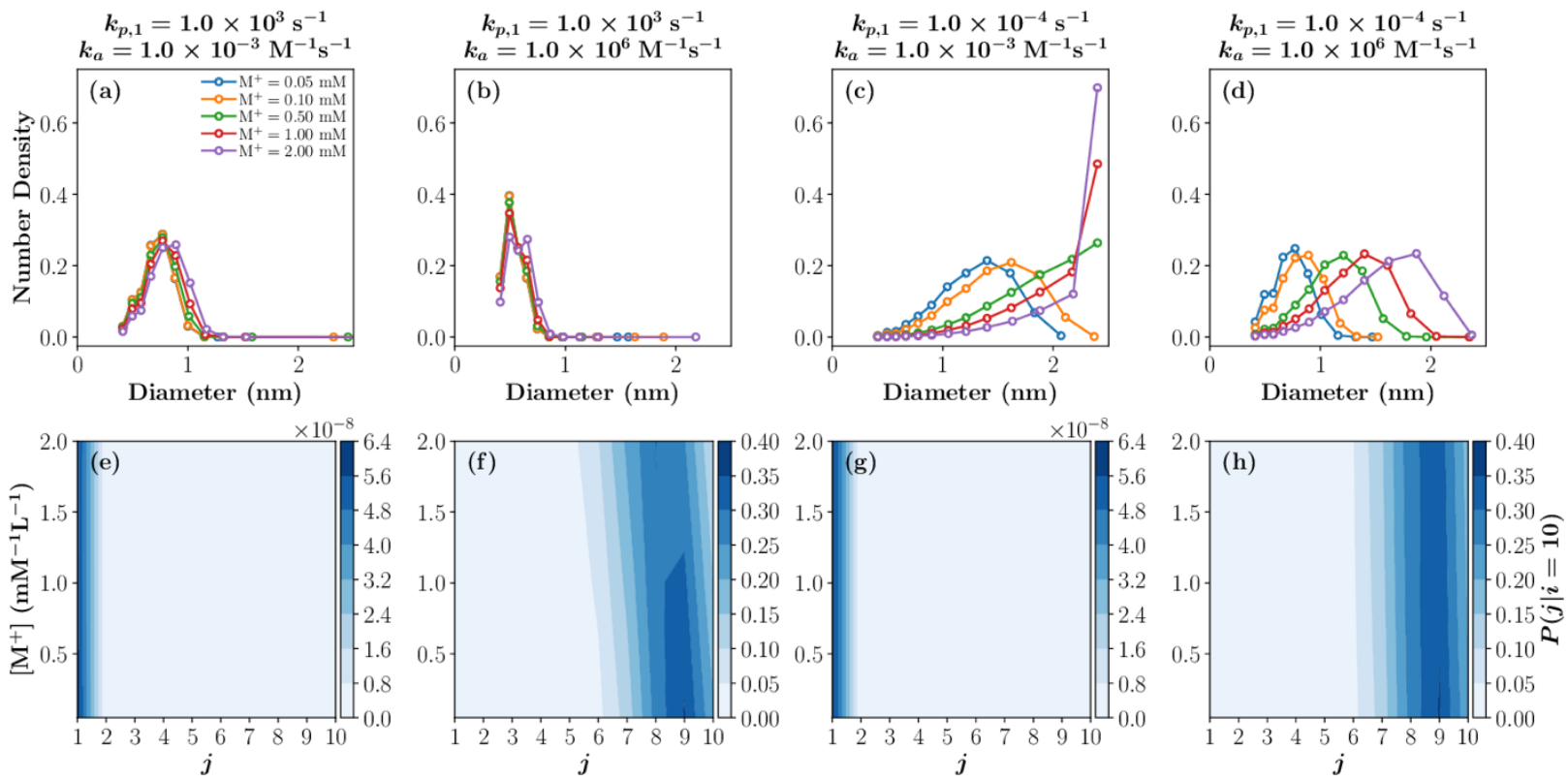

Figure S2: Figure 7 of the manuscript reproduced with a ligand binding rate of $k_{b}=10^{2} \mathrm{M}^{-1} \mathrm{~s}^{-1}$. 



Figure S3: Figure 7 of the manuscript reproduced with a ligand binding rate of $k_{b}=10^{3} \mathrm{M}^{-1} \mathrm{~s}^{-1}$.

Scheme 4: Reaction scheme for Figure S4 incorporating bare metal growth. 


$$
\begin{aligned}
& \mathrm{M}^{+} \stackrel{k_{p, 1}}{\longrightarrow} \mathrm{M} \\
& \mathrm{M}+\mathrm{L} \underset{k_{u b, 1}}{\stackrel{k_{b, 1}}{\rightleftharpoons}} \mathrm{ML} \\
& \mathrm{M}^{+}+\mathrm{L} \underset{k_{u b, 2}}{\stackrel{k_{b, 2}}{\rightleftharpoons}} \mathrm{ML} \\
& \mathrm{ML}^{+} \stackrel{k_{p, 2}}{\longrightarrow} \mathrm{ML} \\
& \mathrm{M}+\mathrm{M} \stackrel{k_{n 1}}{\longrightarrow} \mathrm{C}_{2,0} \\
& \mathrm{ML}+\mathrm{M} \stackrel{k_{n 2}}{\longrightarrow} \mathrm{C}_{2,1} \\
& \mathrm{ML}+\mathrm{M}^{+} \stackrel{k_{n 3, a c}}{\longrightarrow} \mathrm{C}_{2,1} \\
& \mathrm{M}+\mathrm{ML}^{+} \stackrel{k_{n 4, a c}}{\longrightarrow} \mathrm{C}_{2,1} \\
& \mathrm{ML}+\mathrm{ML} \stackrel{k_{n}}{\longrightarrow} \mathrm{C}_{2,2} \\
& \mathrm{ML}+\mathrm{ML}^{+} \stackrel{k_{n, \alpha c}}{\longrightarrow} \mathrm{C}_{2,2} \\
& \mathrm{C}_{i, j}+\mathrm{M} \underset{k_{d 1, j}}{\stackrel{k_{g 1, i, j}}{\rightleftharpoons}} \mathrm{C}_{i+1, j} \\
& \mathrm{C}_{i, j}+\mathrm{M}^{+} \frac{k_{g 2, i, j, a c}}{k_{d 2, j, a c}} \mathrm{C}_{i+1, j} \\
& \mathrm{C}_{i, j}+\mathrm{ML} \underset{k_{d, j+1}}{\stackrel{k_{g, i, j}}{\rightleftharpoons}} \mathrm{C}_{i+1, j+1} \\
& \mathrm{C}_{i, j}+\mathrm{ML}^{+} \frac{k_{g, i, j, a c}}{\underset{k_{d, j+1, a c}}{\rightleftharpoons}} \mathrm{C}_{i+1, j+1} \\
& \mathrm{C}_{i, j}+\mathrm{L} \underset{k_{e, j+1}}{\stackrel{k_{a, i, j}}{\rightleftharpoons}} \mathrm{C}_{i, j+1}
\end{aligned}
$$
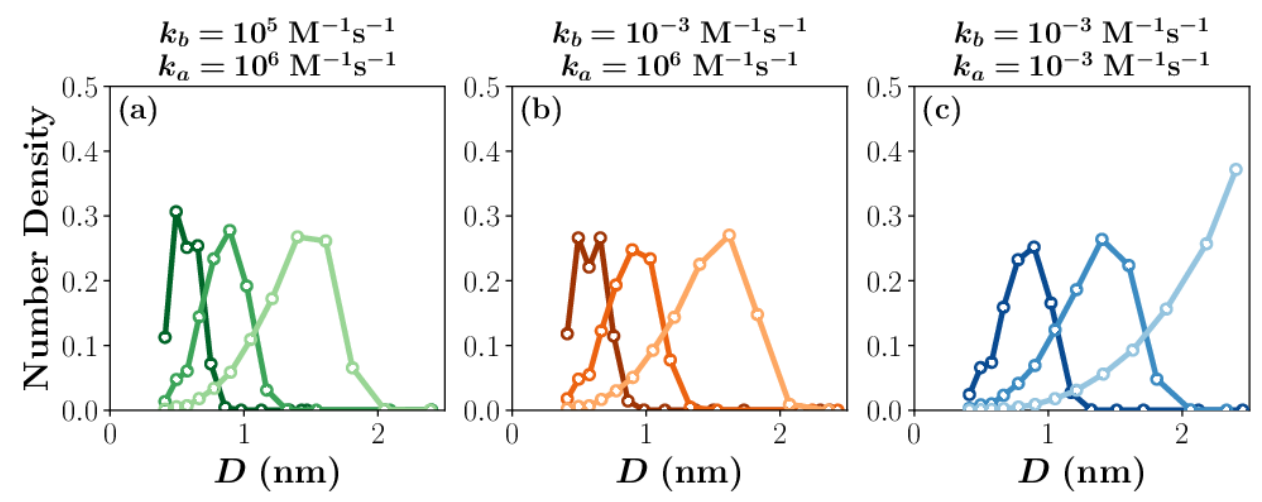

Figure S4: Size distribution for different growth rates (colors) and ligand binding rates: (a) $k_{b}=10^{5} \mathrm{M}^{-1} \mathrm{~s}^{-}$ ${ }^{1}, k_{a}=10^{6} \mathrm{M}^{-1} \mathrm{~s}^{-1}$, (b) $k_{b}=10^{-3} \mathrm{M}^{-1} \mathrm{~s}^{-1}, k_{a}=10^{6} \mathrm{M}^{-1} \mathrm{~s}^{-1}$, and (c) $k_{b}=10^{-3} \mathrm{M}^{-1} \mathrm{~s}^{-1}, k_{a}=10^{-3} \mathrm{M}^{-1} \mathrm{~s}^{-1}$. Dark is $k_{g}$ $=10^{3}$, medium is $10^{4}$, and light is $10^{5} \mathrm{M}^{-1} \mathrm{~s}^{-1}$. The nanoclusters grow uncontrollably as $k_{g}$ increases when the ligand binding rates are small (c). The values for the rate coefficients not listed are the same as listed in Table 2, Scheme 3 in the main text.

\section{References}


1. Lazzari, S.; Theiler, P.; Shen, Y.; Coley, C.; Stemmer, A.; Jensen, K. Ligand-Mediated Nanocrystal Growth. Langmuir 2018, 34 (10), 3307-3315. 